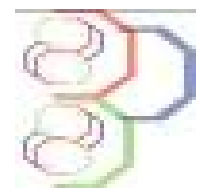

\title{
Perception de la diversité, des choix et de l'aptitude à la transformation des écotypes de maïs cultivés au Bénin
}

\author{
Fresnellia S. Y. Sagboa, M. Vahid Aïssia,b, Alexandre Dansic, Mohamed M. Soumanoua \\ a Unité de Recherche en Génie Enzymatique et Alimentaire, Laboratoire d'Étude et de Recherche en Chimie Appliquée, \\ École Polytechnique d'Abomey-Calavi, Université d'Abomey-Calavi, 01 BP 2009 Cotonou, Bénin. \\ b École des Sciences et Techniques de Conservation et de Transformation des Produits Agricoles, Université \\ d'Agriculture de Kétou, BP 114 Sakété, Bénin. \\ c Laboratoire de Biotechnologies, Ressources Génétiques et Amélioration des Espèces Animales et Végétales, Faculté \\ des Sciences et Techniques de Dassa, Université Polytechnique d'Abomey, BP14 Dassa-zoumé, Benin \\ Auteur correspondant email : msoumanoufr@yahoo.fr
}

Original submitted in on $12^{\text {th }}$ June 2016. Published online at www.m.elewa.org on 31st August 2016

http://dx.doi.org/10.4314/jab.v104i1.12

\section{RÉSUMÉ}

Objectifs : Plusieurs écotypes de maïs sont actuellement cultivés et transformés dans diverses localités au Bénin. Afin de mieux les valoriser, cette étude vise à établir leur répertoire et rapporter les critères de choix de leur production et d'appréciation de leur aptitude aux différentes transformations par les populations locales. Méthodologie et résultats : 242 producteurs et 350 transformateurs de maïs ont été interrogés à travers une enquête menée dans huit zones de forte production du maïs. Des quinze critères de choix des écotypes de maïs cultivés rapportés par les producteurs de maïs, le rendement de production et la commande avec des scores respectifs de 2,64 $\pm 0,06$ et 2,17 $\pm 0,08$ étaient les plus considérés. 12 écotypes améliorés, 51 écotypes locaux et 54 produits alimentaires dérivés ont été répertoriés. Les plus utilisés des dix critères physiques d'appréciation de la qualité des grains ont été le rendement à la mouture $(2,03 \pm 0,55)$ et la grosseur $(1,95 \pm 0,55)$.

Conclusion et application: Cette enquête a permis d'identifier et d'hiérarchiser les critères physiques d'appréciation de la qualité des grains de maïs et les écotypes de maïs utilisés au Bénin. Elle a en outre révélé que les plus aptes à la transformation sont les écotypes locaux. Ces résultats contribuent à une meilleure connaissance de la diversité des écotypes de maïs en usage au Bénin et à une meilleure compréhension de leur choix par les producteurs et les transformatrices.

Mots clés : Zea mays, production, qualité des grains, produits alimentaires, Afrique de l'ouest. 


\section{Sagbo et al. J. Appl. Biosci. 2016 Perception de la diversité, des choix et de l'aptitude à la transformation}

des écotypes de maïs cultivés au Bénin

Perception of the diversity, choices and aptitude for the transformation of the maize ecotypes grown in Benin

Abstract

Objective: Several ecotypes of maize are currently cultivated and processed in various zones of Benin. In order to better valorize them, this study aims to establish their repertory and to report the selection criteria of their production and appreciation of their abilities to be transformed by the local populations.

Methodology and results : 242 maize producers and 350 processors were questioned through a survey carried out in eight zones of strong production of maize. From the 15 selection criteria of the ecotypes of maize cultivated reported by the producers, the yield of production and the order with respective scores of $2.64 \pm 0.06$ and 2.17 \pm 0.08 were used. Twelve improved, fifty-one local ecotypes and fifty-four derived foods were counted. The most used of the ten physical criteria of appreciation of the quality of the grains was the output of grinding $(2.03 \pm 0.55)$ and the size $(1.95 \pm 0.55)$.

Conclusion and application: This investigation helps to identify and to organize into a hierarchy the physical criteria of appreciation of the quality of the maize grains and the ecotypes of maize used in Benin. It moreover revealed that the local ecotypes are most suited to the transformation. These results contribute to a better knowledge of the ecotypes of maize used in Benin and a better comprehension of their choice by the producers and the transformers.

Key words : Zea mays, production, quality of the grains, food products, West Africa.

\section{INTRODUCTION}

La croissance démographique des pays Africains induit une augmentation des besoins alimentaires et nutritionnels mondiaux. La satisfaction de ces besoins et l'essor qualitatif des productions agricoles et agroalimentaires sont devenus les défis permanents auxquels font face actuellement les pays en développement (OCDE, 2011). Le secteur des transformations locales reste alors une opportunité pour assurer la disponibilité des aliments et faciliter l'accès des consommateurs à la nourriture. Par ailleurs, les céréales constituent la base de l'alimentation des populations africaines (Nout et al., 2003) et celle des béninois en particulier (Nago, 1997). L'enjeu de la transformation des céréales est donc fondamental pour le Bénin. En effet, au Bénin, 1. 691.862 tonnes de céréales ont été produites en 2013 (FAO, 2015). Les plus fréquemment utilisées sont le maïs et le sorgho (Baco et al., 2010).. Les céréales, en particulier le maïs sont une véritable source de nutriments dans l'alimentation humaine des pays Africains (Asiedu et al., 1992). L'importance du maïs provient des multiples formes de transformation qu'il subit, qu'elle soit traditionnelle ou industrielle. La transformation du maïs est un domaine occupé essentiellement par les femmes (dans plus de 95\% des cas) notamment dans les régions du Sud et du Centre du Bénin où le maïs représente la principale culture de base pour l'alimentation (PRESAO, 2011). Néanmoins certains postes sont occupés par des hommes (transporteurs, meuniers, producteurs). Les principaux produits locaux obtenus sont la pâte de maïs, l'akassa, le mawè, l'ogui et la bouillie (PRESAO, 2011). Au Bénin, nous avons une multitude de produits dérivés à savoir: la pâte communément appelé "Owô», l'akassa communément appelé «Ogui», le «Akpan» qui est un «yaourt végétal» préparé traditionnellement au Bénin aussi à partir du maïs (Mestres et al., 2001), le «Tchapalo » et le " Adoyo » qui sont des boissons traditionnelles béninoises. Ces produits de grande consommation sont artisanalement préparés et vendus, quotidiennement, dans les rues du Bénin (Anonyme, 1994). Plusieurs écotypes locaux et améliorés de maïs sont utilisés pour la préparation des différents produits dérivés. Le terme écotype est utilisé pour désigner des variétés qui n'ont pas d'identité génétique précise reconnue et stable et qui ne sont pas enregistrées dans des répertoires officiels. Les exigences des transformateurs et des consommateurs vis-à-vis de la qualité du maïs à utiliser dans la préparation des différents produits 
dérivés sont souvent négligées par les sélectionneurs. Ainsi, les objectifs de cette enquête menée dans les zones de grande consommation ont été de recenser les écotypes locaux et améliorés de maïs cultivés au Bénin, d'explorer les critères de

\section{MATÉRIEL ET MÉTHODES}

Échantillonnage et collecte des données : Le maïs étant cultivé et consommé sur toute l'étendue du territoire béninois, une prospection a été faite dans tous les départements du Bénin. Elle a permis de connaître les zones de forte production et de forte consommation du maïs, d'identifier des écotypes de maïs et de retenir les zones à enquêter. Ainsi, huit zones d'enquête (Figure 1) ont été choisies: trois zones urbaines (Cotonou; Bohicon et Parakou) et cinq zones rurales dont deux au Sud (Comé, et Adjohoun), une au Centre (Djidja) et deux au Nord (Djougou et N'dali). L'enquête a été menée de juin 2014 à juillet 2014 sur la base d'un questionnaire choix de leur culture par les producteurs, les critères d'appréciation de la qualité des grains de maïs pour la fabrication des différents produits dérivés et enfin les perceptions sur l'aptitude des différents écotypes à conservation et à la transformation.

adressé aux producteurs de maïs dans les zones rurales et d'un autre adressé aux transformateurs dans les zones urbaines et rurales. Le critère de choix des personnes enquêtées était la connaissance d'au moins trois écotypes de maïs. Ainsi, 92 producteurs ont été enquêtés dans le Sud, 60 dans le Centre et 90 dans le Nord du Bénin, soit au total 242 producteurs généralement des hommes $(91,32 \%)$. Un total de 350 transformateurs majoritairement des femmes $(97,71 \%)$ dont 145 transformateurs dans le Sud, 115 dans le Centre et 90 dans le Nord du Bénin ont été enquêtés.

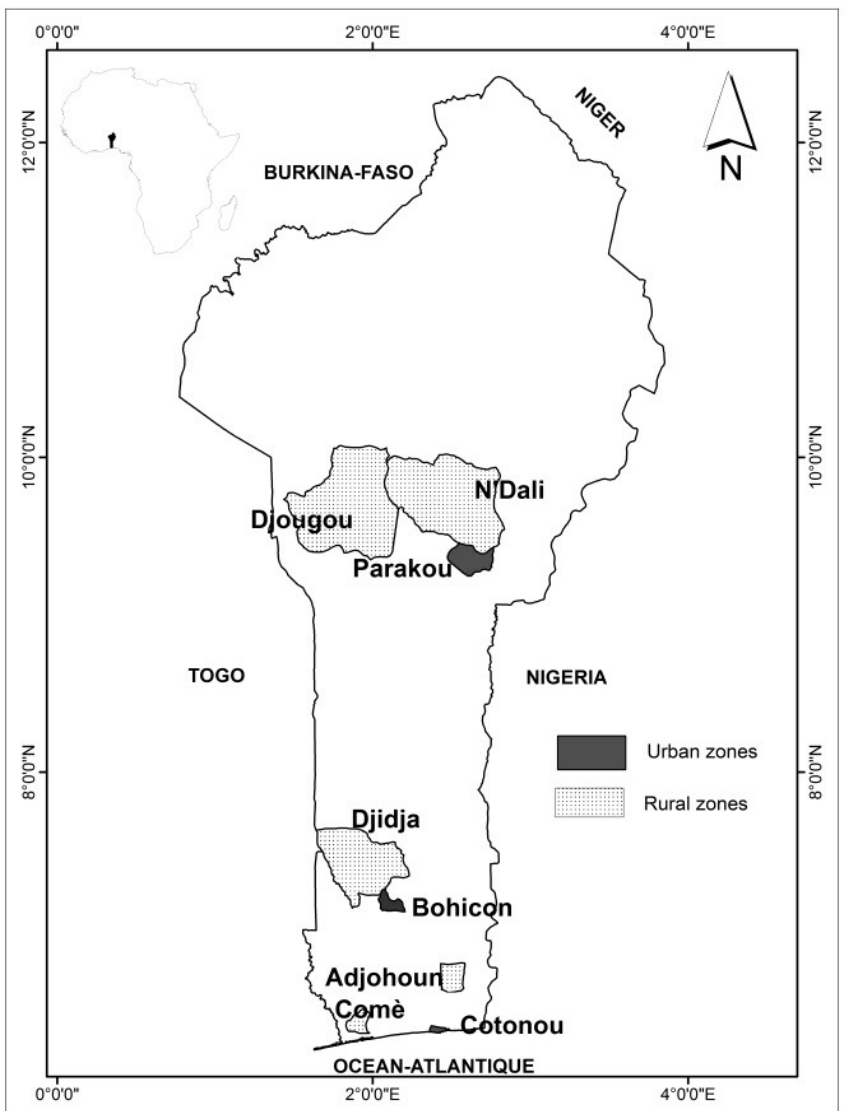

Figure 1 : Les différentes zones enquêtées 
Les données collectées concernent les différents écotypes de maïs produits et utilisés; les critères de choix de leur production, les perceptions sur leur aptitude à la conservation et à la transformation; les critères physiques d'appréciation de la qualité des grains et leur importance ; les produits dérivés de maïs consommés ; leur fréquence d'utilisation et une brève description de leur mode de production.

Analyse statistique: Les fiches remplies ont été dépouillées et les données recueillies analysées à l'aide du logiciel R (R Core development Team, 2013). La technique du scoring a été utilisée et le seuil de signification a été fixé à $\mathrm{a}=0,05$. Elle permet d'évaluer par une note ou un score la probabilité qu'un individu réponde

\section{RÉSULTATS ET DISCUSSION}

Critères de choix des écotypes de maïs cultivés au

Bénin : Quinze critères (Figure 2) sont utilisés par les producteurs de maïs pour le choix des écotypes cultivés. Les critères les plus considérés étaient le rendement de production $(2,64 \pm 0,06)$ et la commande de maïs $(2,17 \pm 0,08)$. La facilité de la mouture des grains $(0,69 \pm 0,07)$, la tolérance à la sécheresse $(0,57 \pm 0,07)$, la durée du cycle $(0,67 \pm 0,08)$, l'aptitude à la conservation des grains $(0,41 \pm 0,07)$, la saison $(0,36 \pm 0,06)$ et la disponibilité d'espace de culture $(0,29 \pm 0,06)$ étaient moins considérés. Les sept critères restants à savoir : le niveau de consommation, la préférence, la disponibilité à une sollicitation ou appartienne à la cible recherchée. Dans cette étude, une échelle variant de 0 à 3 a été retenu. Afin de distinguer les produits dérivés du maïs selon les écotypes de maïs les plus aptes aux transformations, la crédibilité (CRD) des perceptions des répondants de chaque zone sur l'aptitude de chacun des écotypes aux diverses transformations recensées a été déterminée selon Friedman (1986). Le package FactoMineR de Husson et al. (2013) a été utilisé pour l'analyse factorielle de correspondance (AFC) afin d'analyser les perceptions des personnes enquêtées sur l'aptitude de divers écotypes de maïs à être transformés en des produits dérivés avec des niveaux de consommation différents.

des semences, la grosseur des grains ou des épis, l'état sanitaire et la couleur des grains étaient très peu considérés. Les critères identifiés au cours de ce travail confirment les trois premiers critères qui guident le choix des producteurs rapportés par Baco et al. (2010) à savoir respectivement le potentiel de rendement, le cycle cultural et la résistance à la sécheresse. $A$ ces critères rapportés par les producteurs de maïs en zone de savane sèche, s'ajoute spécialement la commande de maïs mentionnée par les producteurs au cours de la présente enquête.

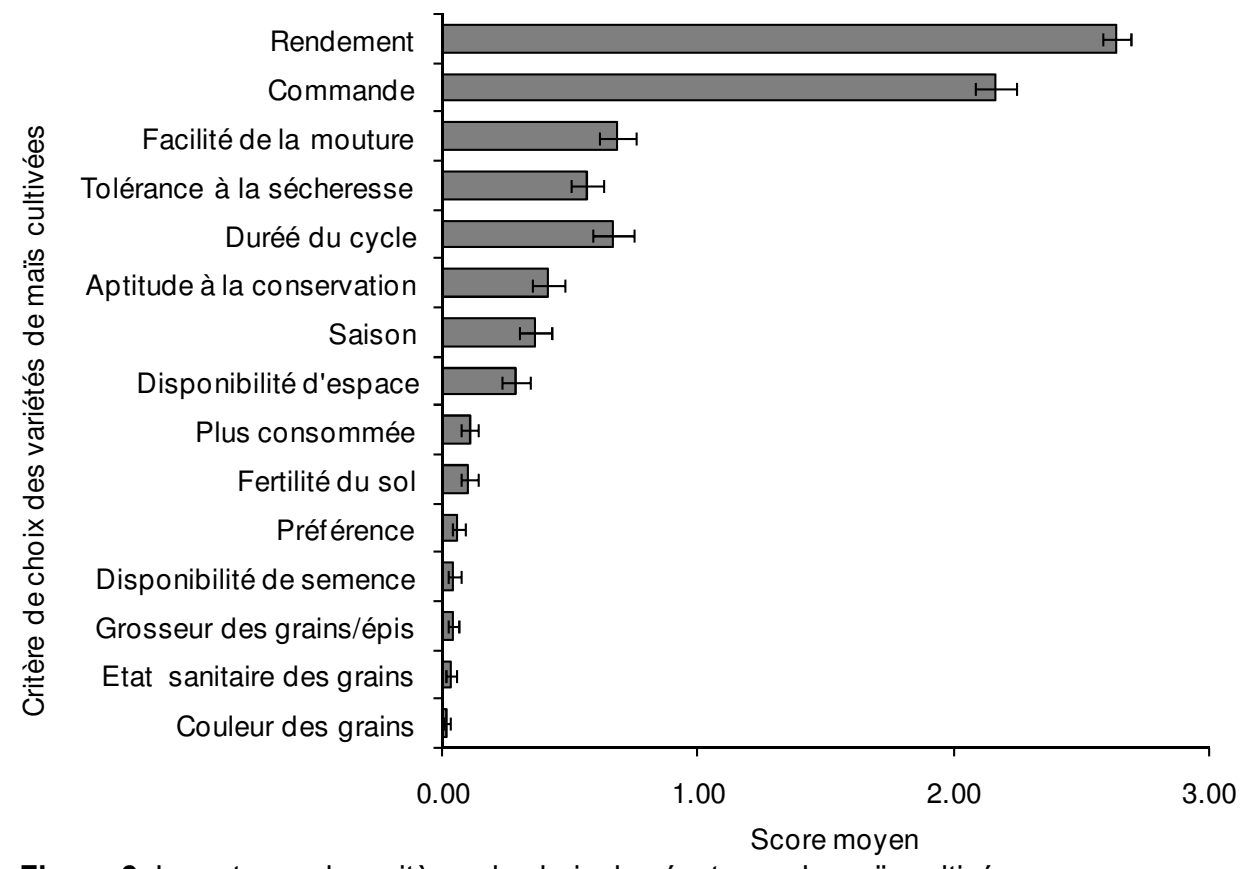

Figure 2. Importance des critères de choix des écotypes de maïs cultivés. 
Diversité des écotypes de maïs cultivés et utilisés au

Bénin : Le nombre d'écotypes de maïs répertoriés varie d'une zone à une autre : quatre à Parakou, six à N'Dali, 18 à Comè. Les autres zones comptaient 10 à 16 écotypes. Au total, 63 écotypes dont 12 améliorés et 51 locaux ont été dénombrés (Tableau 1). Nago et al. (1997) ont étudié 21 écotypes dont 5 locaux et 16 améliorés. Yallou et al. (2010) ont étudié et vulgarisé 15 écotypes améliorés. Parmi ces derniers, sept ont été reconnus et sont utilisés par les personnes enquêtés. De façon générale, trois ou quatre écotypes sont largement connus des répondants quelle que soit la zone, et dans quatre zones (Adjohoun, Bohicon, Comè, Djidja) sur les huit étudiées. La forte richesse variétale observée indiqué par le nombre total d'écotypes dénombrés (Tableau 1) traduit la forte utilisation du maïs dans ces zones du Bénin où le maïs est plus consommé et transformé. Ceci corrobore l'observation faite par Baco et al. (2010), à savoir que le maïs est devenu la céréale dominante en zone de savane sèche au Bénin au regard des superficies emblavées dans six villages enquêtés dont Tiélé, N'Dahonta et Tanongou à Tanguiéta et Sonsoro, Angaradebou puis Sam à Kandi. . II a été remarqué au cours de cette enquête que les producteurs nomment les écotypes selon la région, selon leur cycle végétatif et suivant la dénomination du maïs dans la localité. Ceci peut entraîner l'existence de plusieurs doublons parmi les écotypes dénombrés.

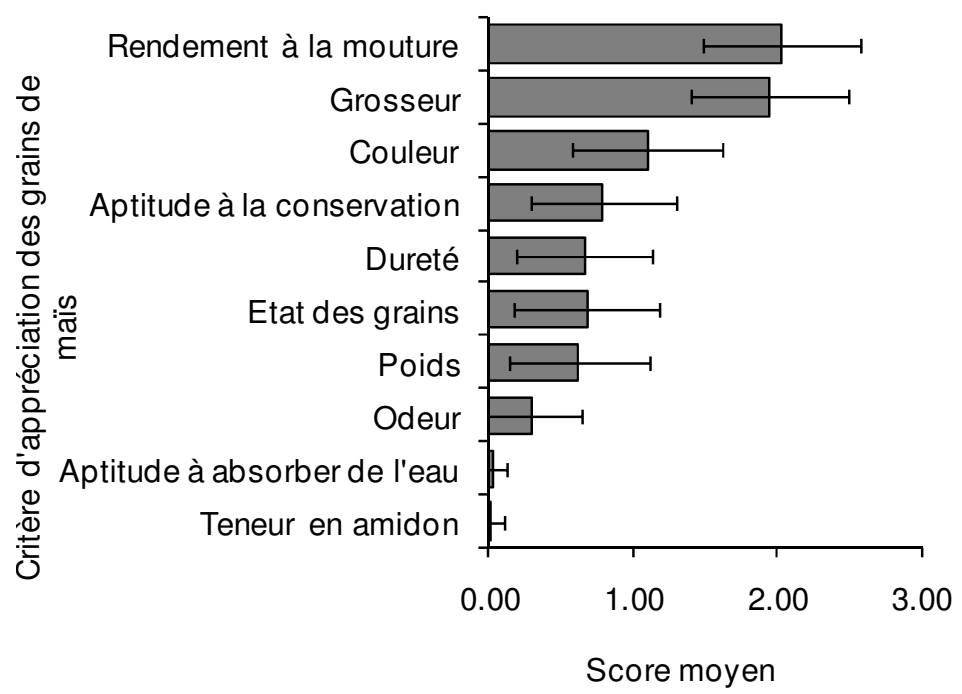

Figure 3. Importance des critères physiques d'appréciation de la qualité des grains de maïs.

Critères physiques d'appréciation de la qualité des grains de maïs : Dix critères physiques d'appréciation de la qualité des grains de maïs ont été rapportés (Figure 3). Les critères les plus utilisés indiqués par le score moyen sont le rendement à la mouture $(2,03 \pm 0,55)$, la grosseur $(1,95 \pm 0,55)$ et la couleur des grains $(1,10 \pm 0,52)$. La dureté, l'état des grains, le poids et l'odeur sont globalement peu considérés (score moyen <1). L'aptitude des grains de maïs à absorber l'eau et la teneur en amidon sont des critères moins considérés (score moyen
$<0,1)$. Les consommateurs et transformateurs ont conscience que la qualité des produits dérivés du maïs dépendent bien des caractéristiques des grains de maïs utilisés. Le rendement à la mouture élevé considéré dans ces critères corrobore les résultats de Nago et al. (1997) qui ont trouvé que les utilisateurs du maïs préfèrent les écotypes locaux présentant des grains tendres et farineux, aux écotypes améliorés souvent à grains durs et vitreux. 
Tableau 1. Écotypes de maïs répertoriés dans les zones rurales et urbaines enquêtées

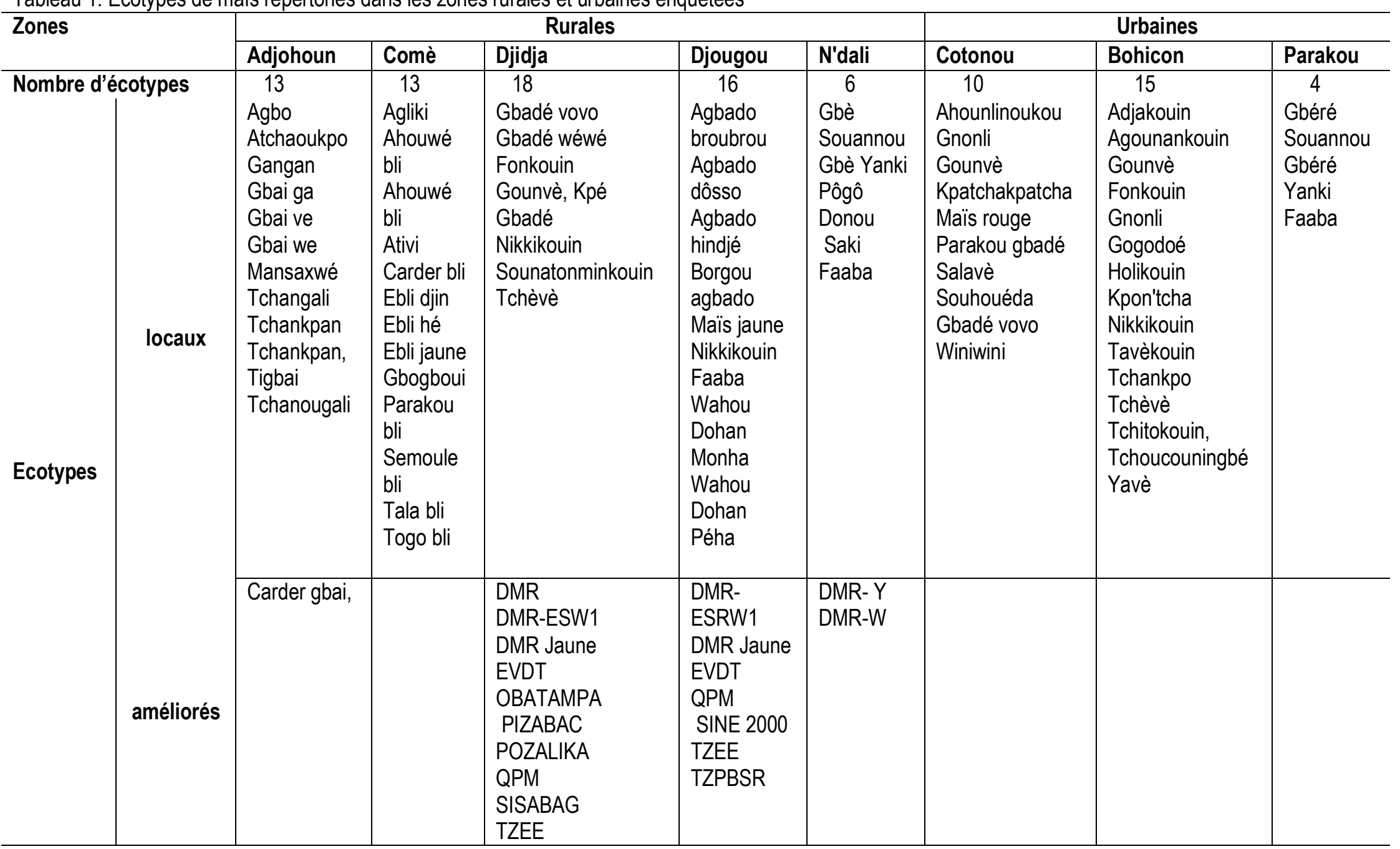


Produits dérivés du maïs et niveau de consommation: Cinquante-quatre (54) produits alimentaires dérivés du maïs ont été répertoriés. Cette valeur est largement supérieure à la quarantaine de recettes à base de maïs obtenues par Nago en 1989 au Bénin. Près du tiers des produits (17 soit 31,48\%) ne sont significativement consommés dans aucune des zones (score de consommation $\approx 0 ; P>0,05$ ). II s'agit de Gbadé dida, Tomintouhui, Kpayè, Atchidemain, Jogoli, Kowé, Hlinhia, Bokounmimin, Dakoï, Liha, Gbailessi, Blikoli, Pain, Klakla, Kokoui, Tchobokoun et Konlime. Parmi les produits significativement consommés, on peut distinguer selon le niveau de consommation (Figure 4), huit produits de grande consommation, dix produits moyennement consommés et 18 produits
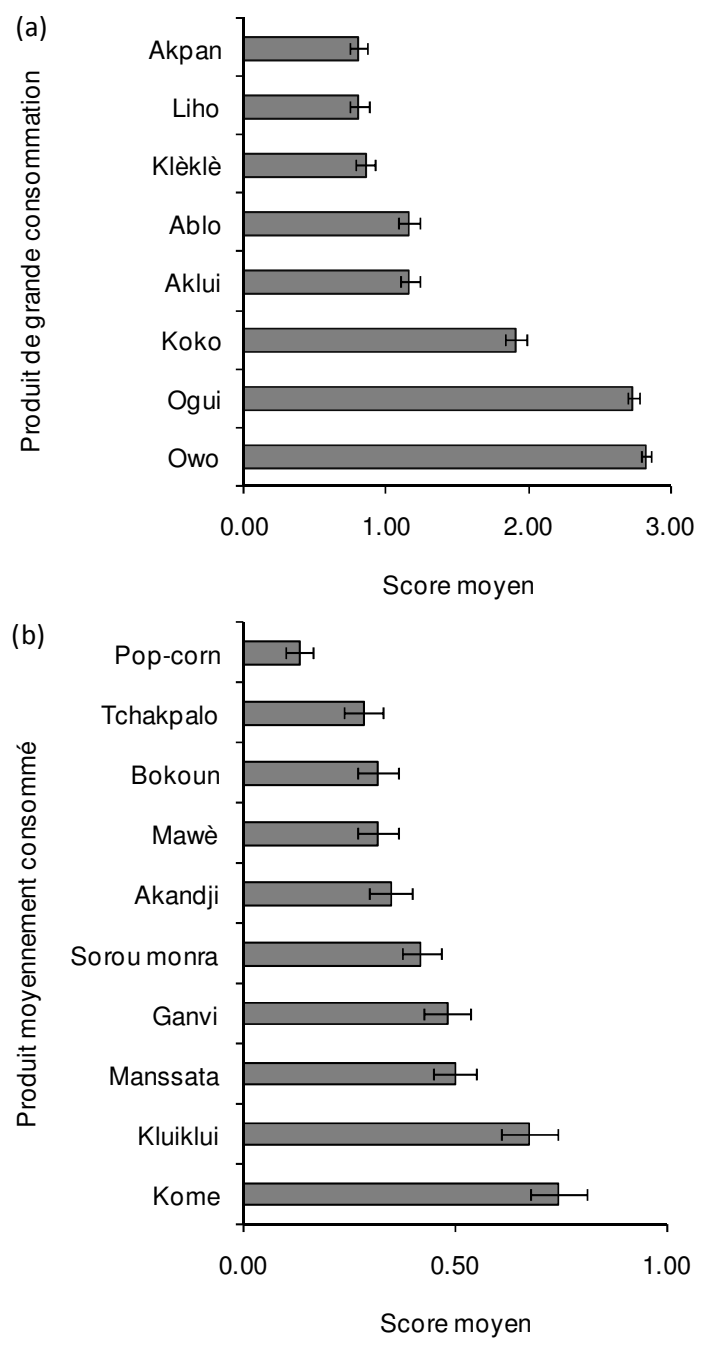

occasionnellement consommés dans au moins une zone. Les produits dérivés du maïs de grande consommation (Figure 4a) sont: Owo, Ogui, Koko, Aklui, Ablo, Klèklè, Liho et Akpan. Ceux moyennement consommés (Figure 4b) sont : Kome, Kluiklui, Manssata, Ganvi, Sorou monra, Akandji, Mawè, Bokoun, Tchakpalo et Pop-corn. Aboété, Abla, Gbê wagué, Yèkè yèkè, Gowé, Kotogba, Gbangba, Gbadémimin, Adjagbé, Kiarou, Tchoukoutou, Don'gba, Kpo, Djikoumé, Adalou, Amiwo, Zankpiti et Simelogoé représentent ceux occasionnellement consommés (Figure 4c). Certains de ces produits ont été brièvement décrits (Tableau 2). Notons que ces différentes transformations et utilisations du maïs sont plus remarquées dans le Sud du Bénin.

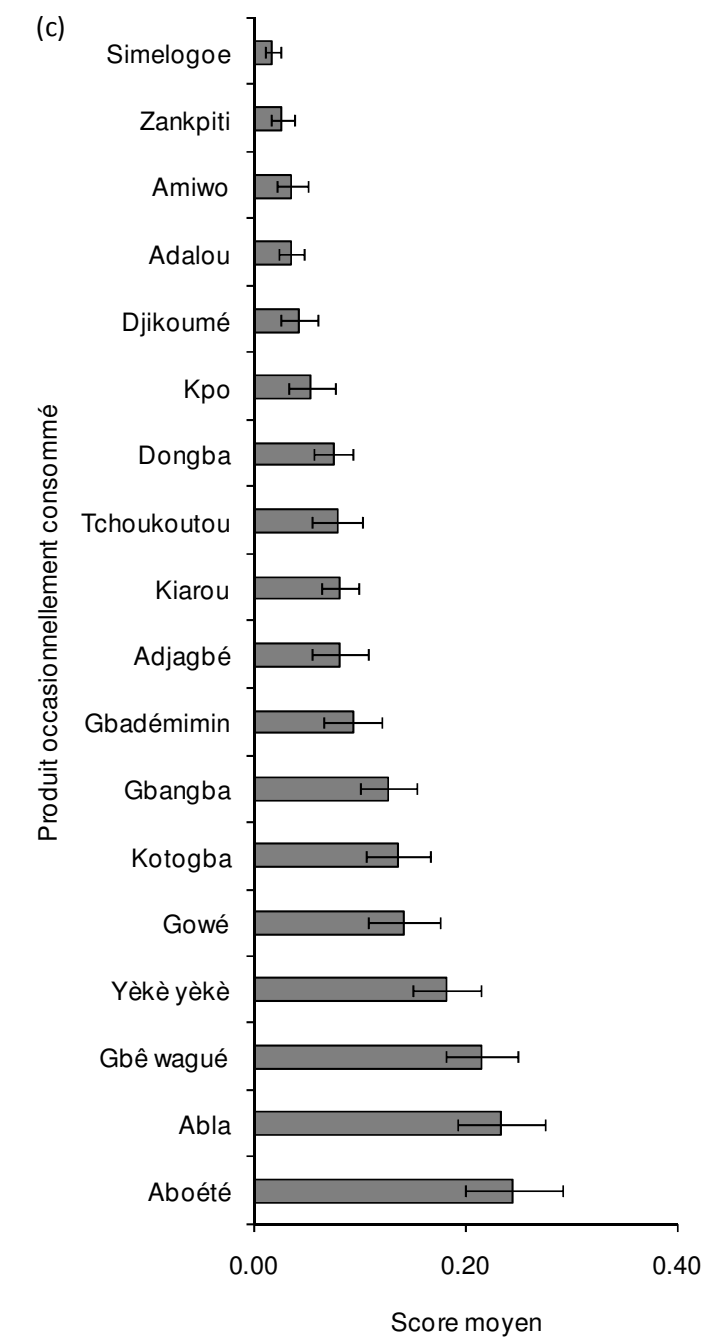

Figure 4 : Niveau de consommation des produits dérivés du maïs 


\section{Sagbo et al. J. Appl. Biosci. 2016 Perception de la diversité, des choix et de l'aptitude à la transformation}

des écotypes de maïs cultivés au Bénin

Tableau 2 : Description sommaire de quelques produits dérivés du maïs

\begin{tabular}{l|l}
\hline Produits & Description sommaire \\
\hline Akpan & Yaourt végétal à base de maïs fermenté \\
\hline Liho & Pâte dure à base de maïs fermentée \\
\hline Klèklè & Galette à base de maïs et condiments \\
\hline Ablo & Gâteau cuit au bain marie de maïs et de riz \\
\hline Aklui & Bouillie de mawè roulé \\
\hline Koko & Bouillie à base de farine de maïs \\
\hline Pop corn & Maïs soufflé \\
\hline Tchakpalo & Boissons à base de malt de maïs \\
\hline Bokoun & Grains de maïs bouillis avec de l'arachide \\
\hline Mawè & Farine fermentée de maïs \\
\hline Akandji & Pâte sucrée à base de maïs emballée dans des feuilles de banane \\
\hline Sorou monra & Bouillie à base de farine de maïs et de la potasse (spécialité du Nord Bénin) \\
\hline Simelogoe & Pâte sucrée à base de maïs \\
\hline Zankpiti ou djongoli & Haricot et farine de maïs \\
\hline Amiwo & Pâte rouge assaisonnée de maïs \\
\hline Manssata & Gâteau sucrée à base de farine de maïs légèrement fermentée \\
\hline Djikoumè & Pâte assaisonnée de maïs \\
\hline Yèkè-yèkè & Couscous de maïs \\
\hline Gbè wagué ou gbadémimin & Epis de maïs grillé \\
\hline Abla & Pâte à base de maïs et de haricot cuite à la vapeur \\
\hline Aboété & Pâte fermentée sucrée de maïs consommé comme boisson \\
\hline Klakou & Galette de maïs assaisonnée \\
\hline Adjagbè & $\begin{array}{l}\text { Bouillon alimentaire cuit à base de farine de maïs (petite quantité) et les légumes } \\
\text { feuilles ou de manioc emballée dans des feuilles de banane }\end{array}$ \\
\hline
\end{tabular}

Perceptions de l'aptitude des différents écotypes de maïs à la transformation en divers produits dérivés : Les perceptions de l'aptitude des écotypes de maïs à la transformation ont été renseignées pour I divers produits répertoriés en fonction du niveau de consommation (Figure 5). La crédibiilité de ces perceptions a été évalué pour les produits bien représentés sur les AFC (Tableau 3) et pour ceux n'apparaissant pas sur les AFC (Tableau 4). Ces perceptions sont importantes (CRD $\geq 5,00 \%$ ) pour les 55 écotypes répertoriés sur l'ensemble des 8 zones enquêtées et crédibles (CRD $\geq 50,00 \%$ ) sur 22 de ces écotypes pour au moins un produit. II s'agit de Adjakouin et Nikkikouin à Bohicon, Ahouwébli à Comè, EVDT à Djidja, Saki faaba à Parakou et à N'Dali, Gbéré yanki à N'Dali. Les deux premiers axes de l'AFC (Figure 5a) réalisé sur les perceptions de l'aptitude des écotypes aux transformations en produits dérivés de grande consommation expliquaient $54,24 \%$ de la variation totale. Les produits dérivés Ogui, Owo, Mawè et Liho n'apparaissaient sur aucun de ces axes factoriels. Ceci explique que ces trois produits très consommés peuvent être préparés à partir de n'importe quels écotypes de maïs. Les études menées par Nago et al. (1997) ont révélé que les écotypes locaux Gnonli et Gbaévè pourraient être considérés comme plus aptes à la préparation de la pâte "Owo ». Les perceptions de l'aptitude des écotypes de maïs à la transformation ont été renseignées pour tous les produits dérivés moyennement consommés (Figure $5 b$ ) sauf le Pop corn qui n'est pas un produit local. Les perceptions sont importantes (CRD $\geq 5,00 \%$ ) pour 36 écotypes sur l'ensemble des 8 zones enquêtées et crédibles (CRD $\geq$ $50,00 \%$ ) sur six de ces écotypes pour au moins un produit. Seul l'écotype Saki faaba a une aptitude crédible $(C R D \geq 5,00 \%)$ à Parakou pour à la fois deux produits moyennement consommés (Manssata et Sorou monra) sur les dix produits de cette catégorie. Certains produits moyennement consommés présentaient des similitudes quant aux écotypes de maïs les plus cités comme appropriés à leurs transformations. Ainsi, les écotypes de maïs les plus souvent indiqués pour la transformation en Kluiklui sont souvent cités pour la transformation en 


\section{Sagbo et al. J. Appl. Biosci. 2016 Perception de la diversité, des choix et de l'aptitude à la transformation}

des écotypes de maïs cultivés au Bénin

Ganvi, en Akandji et en Bokoun. Les écotypes communs aux quatre produits sont Adjakouin, Nikkikouin, Fonkouin, et Carderkouin à Bohicon. Les écotypes DMR, EVDT, Gounvè, PIZABAC, POZALIKA et SISABAG (de Djidja) sont les plus cités comme aptes à la transformation en Tchakpalo. Pour la préparation du Kome, les écotypes les plus indiqués sont Agbado broubrou, Borgou agbado et Faaba à Djougou, Carder bli, Ahouwé bli et Parakou blià Comè. Les écotypes les plus souvent indiqués comme aptes à la préparation du Manssata sont Gbéré yanki, Gbéré sounnou et Saki faaba (à N'Dali). Les écotypes Gbéré yanki, Gbéré sounnou, Saki faaba et Gbé kékérékou (à Parakou) étaient les plus souvent indiqués comme aptes à la transformation en Sorou monra. Pour la préparation du Mawè, les écotypes les plus indexés comme aptes sont Adjakouin et Nikkikouin (à Bohicon). Les perceptions de l'aptitude des écotypes de maïs à la transformation ont été renseignées pour 12 produits occasionnellement consommés (Figure 5c). Les perceptions concernant les 12 produits sont importantes (CRD $\geq 50,00 \%$ ) pour 30 écotypes (cumul sur les 8 zones enquêtées), mais aucune des perceptions n'était crédibles (CRD $<50,00 \%$ ). Les perceptions de l'aptitude de l'écotype Adjakouin sont importants (CRD $\geq 50,00 \%$ ) pour à la fois sept produits occasionnellement consommés sur les 12 renseignés dans cette catégorie. Six produits dérivés sur douze sont bien représentés sur au moins l'un de ces axes factoriels. Les six produits n'apparaissant pas sur les AFC sont Adjagbé, Don'gba, Gbadémimin, Gowé, Kpo et Tchoukoutou. Ceci signifie que ces produits dérivés peuvent être fabriqués à partir de plusieurs écotypes de maïs. La projection des écotypes et des produits dérivés du maïs occasionnellement consommés bien représentés dans le plan principal (Figure $5 c$ ) montre que certains produits présentaient des similitudes quant aux écotypes de maïs les plus cités comme appropriés à leurs transformations. Ainsi, pour la préparation du Djikoumé et du Ablo kpomèton, les écotypes les plus indiqués étaient Ahouwé bli, Carder bli, Parakou bli et Ebli jaune (à Comè). De même, pour préparer du Abla, du Aboété ou du Kotogba, les écotypes les plus indiqués étaient Adjakouin, Nikkikouin et Fonkouin à Bohicon. Les écotypes les plus souvent indiqués comme aptes à la préparation du Yèkèyèkè étaient Gbéré yankiet Saki faaba à Parakou et à N'Dali, Gbéré kékérékou à Parakou, Faaba, Agbado broubrou, Agbado dôsso et Borgou agbado à Djougou. Les écotypes les plus souvent indiquées comme aptes à la préparation du Yèkèyèkè étaient Gbéré yanki et Saki faaba à Parakou et à N'Dali, Gbéré kékérékou à Parakou, Faaba, Agbado broubrou, Agbado dôsso et Borgou agbado à Djougou. Les perceptions de l'aptitude des écotypes de maïs à la transformation ont été renseignées pour sept produits rarement consommés.

Tableau 3 : Niveaux de crédibilité des perceptions de l'aptitude à la transformation des écotypes de maïs en divers produits n'apparaissant pas sur les AFC.

\begin{tabular}{|c|c|c|}
\hline Produits & Variétés aptes à la transformation & Crédibilité (CRD) (\%) \\
\hline \multirow{4}{*}{ Owo } & Gbé yanki, Saki faaba, Gbéré sounnou & 100 \\
\hline & DMR, Gbai ve & $95 \leq \mathrm{CRD}<100$ \\
\hline & $\begin{array}{l}\text { EVDT, Nikkikouin, Adjakouin, Saki Faaba, Borgou agbado, Ahouwé bli, } \\
\text { Agbado broubrou, Parakou bli }\end{array}$ & $70 \leq \mathrm{CRD}<90$ \\
\hline & $\begin{array}{l}\text { Carder gbai, Gbai vé, Gbé yanki, POZALIKA, Winiwini, Gbai wé, DMR, Gbê } \\
\text { Kékérékou, Agbado dôsso }\end{array}$ & $40 \leq \mathrm{CRD} \leq 60$ \\
\hline \multirow{3}{*}{ Ogui } & DMR, Gbéré yanki, Saki faaba, & $95 \leq \mathrm{CRD}<100$ \\
\hline & Saki faaba, Nikkikouin, Winiwini, Parakou bli, Borgou agbado & $70 \leq \mathrm{CRD}<90$ \\
\hline & $\begin{array}{l}\text { Agbado broubrou, Gbai vé, Adjakouin, Ahouwé bli, Gbai wé, EVDT, } \\
\text { POZALIKA, Gbé yanki, Carder gbai, POZALIKA }\end{array}$ & $40 \leq \mathrm{CRD} \leq 60$ \\
\hline \multirow[t]{2}{*}{ Liho } & Nikkikouin, Adjakouin, EVDT, DMR, & $40 \leq \mathrm{CRD}<65$ \\
\hline & $\begin{array}{l}\text { Parakou bli, Carder gbai, DMR, Ahouwé bli, PIZABAC, Fonkouin, Gbai vé, } \\
\text { POZALIKA, Winiwini, Kpatchakpatcha, Gbai we, Gounvè, Tchèvè, QPM }\end{array}$ & $5 \leq \mathrm{CRD} \leq 30$ \\
\hline Mawè & Adjakouin, Nikkikouin, Fonkouin, Tchoucouningbé & $5 \leq \mathrm{CRD} \leq 15$ \\
\hline Gowé & Adjakouin, Nikkikouin, Fonkouin, Kpon'tcha & $5 \leq \mathrm{CRD}<15$ \\
\hline Liha & Parakou bli, Ahouwé bli, Carder gbai, Ebli jaune & $5 \leq \mathrm{CRD}<15$ \\
\hline Gbadémimin & DMR, Adjakouin, Nikkikouin ; Gbai we & 5 \\
\hline Kро & Adjakouin, Nikkikouin, Fonkouin, Carder gbai & 5 \\
\hline
\end{tabular}


Sagbo et al. J. Appl. Biosci. 2016 Perception de la diversité, des choix et de l'aptitude à la transformation des écotypes de maïs cultivés au Bénin

\begin{tabular}{|l|l|l|}
\hline Cokoui & Ahouwé bli, Parakou bli, Carder gbai, Gbai ve & 5 \\
\hline Tchoukoutou & Adjakouin, EVDT, POZALIKA & 5 \\
\hline Adjagbé & Fonkouin, Nikkikouin & 5 \\
\hline Blicoli & Ahouwé bli, Carder gbai, Parakou bli & $<5$ \\
\hline $\begin{array}{l}\text { Bocounmimi } \\
\text { n }\end{array}$ & Gnonli & 2.50 \\
\hline Pain & Gbai we & 2.50 \\
\hline
\end{tabular}

Tableau 4 : Niveaux de crédibilité des perceptions de l'aptitude à la transformation des écotypes de maïs en divers produits bien représentés sur les AFC.

\begin{tabular}{|c|c|c|}
\hline Produits & Variétés aptes à la transformation & Crédibilité (CRD) (\%) \\
\hline \multirow[t]{4}{*}{ Koko } & Saki faaba, & $95 \leq \mathrm{CRD}<100$ \\
\hline & POZALIKA, EVDT, & $70 \leq \mathrm{CRD}<90$ \\
\hline & $\begin{array}{l}\text { PORTA, Borgou agbado, Faaba, Agbado broubrou, Adjakouin, Nikkikouin, } \\
\text { Parakou ebli, Carder bli, }\end{array}$ & $40 \leq \mathrm{CRD}<70$ \\
\hline & $\begin{array}{l}\text { DMR, Fonkouin, Kpatchakpatcha, Ebli jaune, Agbado dôsso, Gbawéwé, } \\
\text { Winiwini, POZALIKA, Gbadé vovo }\end{array}$ & $10 \leq \mathrm{CRD}<40$ \\
\hline \multirow[t]{4}{*}{ Ablo } & Gbéré yanki, Saki faaba, & $95 \leq \mathrm{CRD}<100$ \\
\hline & Parakou ebli, DMR & $70 \leq \mathrm{CRD}<90$ \\
\hline & Ahouwé bli, Carder bli, Gbé kékérékou, & $40 \leq \mathrm{CRD}<70$ \\
\hline & $\begin{array}{l}\text { Ebli jaune, Gbéré yanki, PIZABAC, Adjakouin, POZALIKA, Nikkikouin, } \\
\text { Gbawéwé, EVDT, Ebli djin, Winiwini, Ebli hé }\end{array}$ & $10 \leq \mathrm{CRD}<40$ \\
\hline \multirow[t]{4}{*}{ Klèklè } & Gbéré yanki, Saki faaba, & 100 \\
\hline & DMR, Tchèvè, & $70 \leq \mathrm{CRD}<90$ \\
\hline & POZALIKA, Gbéré yanki, Gbé kékérékou, Adjakouin, & $40 \leq \mathrm{CRD}<70$ \\
\hline & $\begin{array}{l}\text { Nikkikouin, Borgou agbado, Agbado broubrou, Fonkouin, Ahouwébli, Carder } \\
\text { bli, Saki faaba, Parakou ebli, Faaba, Ebli jaune, Agbado dôsso, Gbéré } \\
\text { sounnoun }\end{array}$ & $10 \leq \mathrm{CRD}<40$ \\
\hline
\end{tabular}

Tableau 4 : Niveaux de crédibilité des perceptions de l'aptitude à la transformation des écotypes de maïs en divers produits bien représentés sur les AFC (Suite).

\begin{tabular}{|c|c|c|}
\hline Produits & Variétés aptes à la transformation & $\begin{array}{l}\text { Crédibilité (CRD) } \\
(\%)\end{array}$ \\
\hline \multirow[t]{5}{*}{ Aklui } & Gbéré yanki & $95 \leq \mathrm{CRD}<100$ \\
\hline & DMR & $70 \leq \mathrm{CRD}<90$ \\
\hline & POZALIKA, Gbéré yanki, Ahouwébli, Tchèvè, Gbé kékérékou, Gbai wé & $40 \leq \mathrm{CRD}<70$ \\
\hline & $\begin{array}{l}\text { Agbado broubrou, Faaba, Parakou ebli, Gbai wéwé, PIZABAC, Carder bli, } \\
\text { Gbadé vovo }\end{array}$ & $10 \leq \mathrm{CRD}<40$ \\
\hline & Adjakouin, Ebli jaune, Tchangali, SISABAG, Tchèvè & 5 \\
\hline \multirow[t]{3}{*}{ Manssata } & Saki faaba, Gbéré yanki, Gbéré sounnou, & $40 \leq \mathrm{CRD}<70$ \\
\hline & $\begin{array}{l}\text { Gbéré yanki, DMR, Gbéré sounnou, Agbado broubrou, Gbé kékérékou, } \\
\text { POZALIKA, Faaba, Ahouwé bli, Carder bli, Gbawéwé, Agbado dôsso, } \\
\text { Parakou ebli }\end{array}$ & $10 \leq \mathrm{CRD}<40$ \\
\hline & Ebli hé, Maïs jaune & $05 \leq \mathrm{CRD}<10$ \\
\hline \multirow[t]{3}{*}{ Kluiklui } & DMR, Tchèvè, POZALIKA & $40 \leq \mathrm{CRD}<70$ \\
\hline & Nikkikouin, Adjakouin, EVDT, Fonkouin & $10 \leq \mathrm{CRD}<40$ \\
\hline & $\begin{array}{l}\text { Ahouwé bli, Carder bli, DMR, Gbawéwé, POZALIKA, Gounvè, SISABAG, } \\
\text { Parakou ébli, Ebli jaune, PIZABAC, Ebli hé, Maïs jaune, Tavèkouin }\end{array}$ & $05 \leq \mathrm{CRD}<10$ \\
\hline
\end{tabular}




\section{Sagbo et al. J. Appl. Biosci. 2016 Perception de la diversité, des choix et de l'aptitude à la transformation}

des écotypes de maïs cultivés au Bénin

\begin{tabular}{|c|c|c|}
\hline \multirow[t]{3}{*}{ Sorou monra } & Saki faaba, Gbéré yanki & $40 \leq \mathrm{CRD}<70$ \\
\hline & Gbé kékérékou & $10 \leq \mathrm{CRD}<40$ \\
\hline & Gbéré sounnou & $05 \leq \mathrm{CRD}<10$ \\
\hline \multirow[t]{3}{*}{ Kome } & Parakou ébli, Ahouwé bli, Carder bli, & $40 \leq \mathrm{CRD}<70$ \\
\hline & $\begin{array}{l}\text { Saki faaba, Nikkikouin, Agbado broubrou, Faaba, Borgou agbado, } \\
\text { Adjjakouin, Ebli jaune, Gbéré yanki, Gbé kékérékou, Ebli djin, }\end{array}$ & $10 \leq \mathrm{CRD}<40$ \\
\hline & Ebli hé & 5 \\
\hline \multirow[t]{2}{*}{ Akpan } & $\begin{array}{l}\text { Gbai wé, Kpatchakpatcha, EVDT, POZALIKA, Adjakouin, Nikkikouin, } \\
\text { DMR, Winiwini, Gbadé vovo, Agbado broubrou, Faaba, PIZABAC, } \\
\text { Fonkouin }\end{array}$ & $10 \leq \mathrm{CRD}<40$ \\
\hline & $\begin{array}{l}\text { Ebli jaune, Carder gbai, Tchangali, Parakou ebli, Carder bli, Borgou } \\
\text { agbado, Agbo }\end{array}$ & $05 \leq \mathrm{CRD}<10$ \\
\hline \multirow[t]{2}{*}{ Yèkè yèkè } & Saki faaba, Gbéré yanki, Agbado broubrou, Faaba, Borgou agbado & $10 \leq \mathrm{CRD}<40$ \\
\hline & Gbê kékérékou, Agbado dôsso & $05 \leq \mathrm{CRD}<10$ \\
\hline \multirow[t]{2}{*}{ Ganvi } & Nikkikouin, Adjakouin, Fonkouin, EVDT, DMR & $10 \leq \mathrm{CRD}<40$ \\
\hline & Carderkouin, POZALIKA, Tavèkouin & $05 \leq \mathrm{CRD}<10$ \\
\hline \multirow[t]{2}{*}{ Akandji } & Adjakouin, DMR, Nikkikouin, POZALIKA, Tchèvè & $10 \leq \mathrm{CRD}<40$ \\
\hline & Fonkouin, Gbawéwé, Carderkouin & $05 \leq \mathrm{CRD}<10$ \\
\hline \multirow[t]{3}{*}{ Tchakpalo } & Saki faaba, Gbé kékérékou & $40 \leq \mathrm{CRD}<70$ \\
\hline & EVDT, Gbéré yanki, Gbéré sounnou, POZALIKA, DMR, SISABAG & $10 \leq \mathrm{CRD}<40$ \\
\hline & PIZABAC3 (7.50 \%), Gounvè3 (7.50 \%), Ebli jaune2 (5.00 \%) & $05 \leq \mathrm{CRD}<10$ \\
\hline \multirow[t]{2}{*}{ Kotogba } & DMR, Tchèvè, POZALIKA & $10 \leq \mathrm{CRD}<40$ \\
\hline & Adjakouin, Gbawéwé), Maïs jaune & $05 \leq \mathrm{CRD}<10$ \\
\hline Abla & DMR, Tchèvè, Adjakouin, Nikkikouin, POZALIKA, Fonkouin, Gbawéwé & $10 \leq \mathrm{CRD}<40$ \\
\hline \multirow[t]{3}{*}{ Tomintouhoui } & DMR & 40 \\
\hline & Tchèvè, POZALIKA & $10 \leq \mathrm{CRD}<40$ \\
\hline & Adjakouin, Gbawéwé, Carderkouin & $05 \leq \mathrm{CRD}<10$ \\
\hline Bokoun & Adjakouin, Nikkikouin, Fonkouin & $10 \leq \mathrm{CRD}<40$ \\
\hline Dakoï & Saki faaba, Gbéré yanki, Gbé kékérékou, Gbéré sounnou & $10 \leq \mathrm{CRD}<40$ \\
\hline \multirow[t]{2}{*}{ Aboété } & DMR, POZALIKA, Adjakouin, & $10 \leq \mathrm{CRD}<40$ \\
\hline & Nikkikouin, Fonkouin, EVDT, Tchèvè, DMR, Tavèkouin & $05 \leq \mathrm{CRD}<10$ \\
\hline \multirow[t]{2}{*}{ Don'gba } & EVDT, Gounvè, SISABAG & $10 \leq \mathrm{CRD}<40$ \\
\hline & Saki faaba, Gbéré yanki, DMR, Tchèvè, Gbawéwé, POZALIKA & $05 \leq \mathrm{CRD}<10$ \\
\hline Ablo kpometo & Ahouwé bli, Carder bli & 2.50 \\
\hline
\end{tabular}



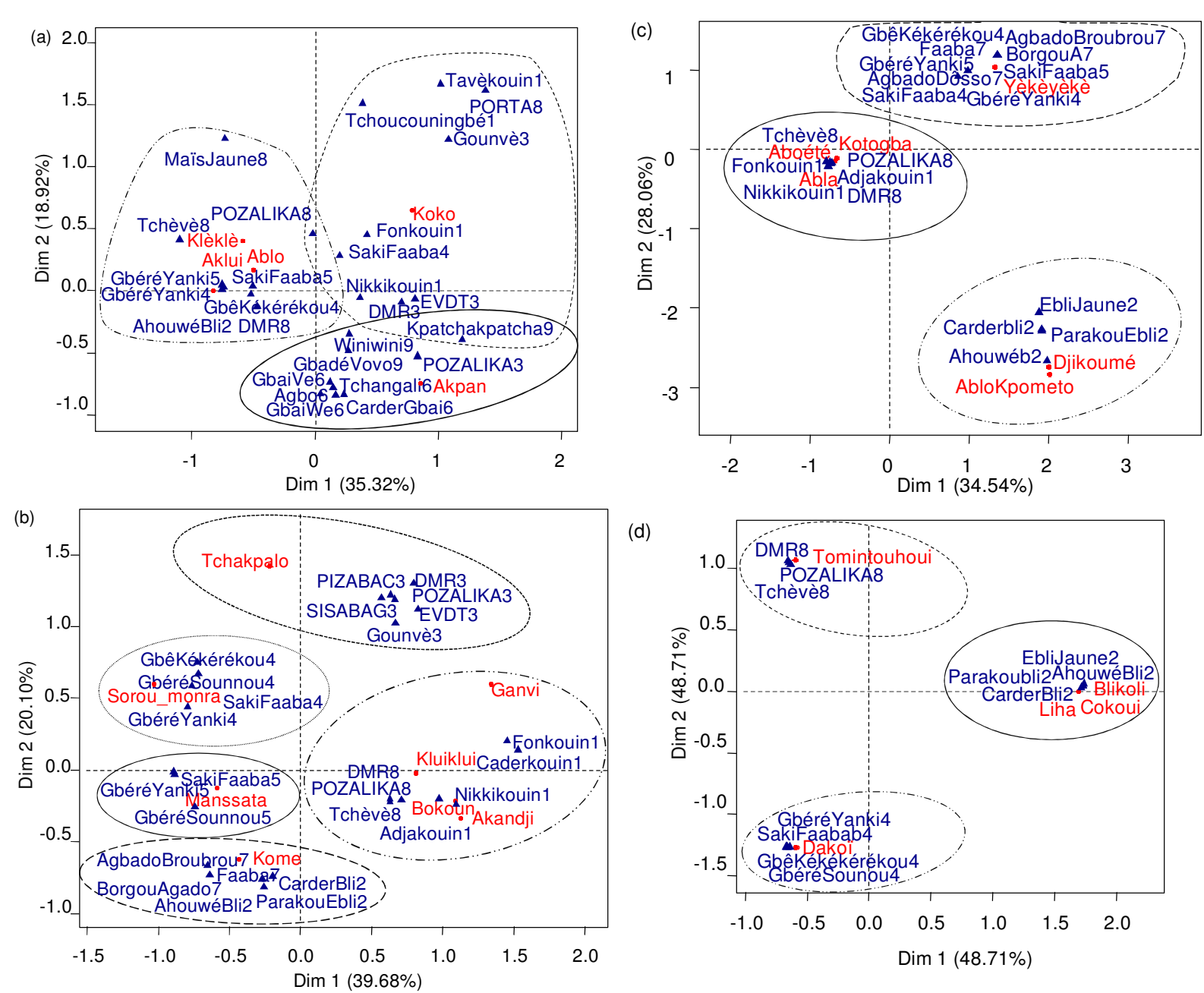

Figure 5. Relations entre écotypes de maïs et produits dérivés de grande consommation (a), moyennement consommés (b), occasionnellement consommés (c) et rarement consommés (d).

Les noms des écotypes et des produits dérivés sont respectivement écrits en bleu et en rouge. Les chiffres devant les noms des écotypes font référence aux zones : Bohicon (1), Comè (2), Djidja (3), Parakou (4), N'Dali (5), Adjohoun (6), Djougou (7) et Cotonou (9).

\section{CONCLUSION}

Le maïs est une céréale très cultivée, consommée et transformée partout au Bénin. La production du maïs est plus destinée aux hommes et sa transformation en divers produits dérivés plus destinée aux femmes. Parmi les 10 critères physiques d'appréciation de la qualité des grains de maïs, les plus considérés sont le rendement à la mouture, la grosseur et la couleur. Selon la dénomination des producteurs et des transformatrices, soixante-trois (63) écotypes dont douze (12) améliorés et cinquante-etun (51) locaux ont été répertoriés. Parmi les quinze critères, utilisés par les producteurs de maïs pour le choix des écotypes cultivés, les plus considérés étaient le rendement de production et la commande ou demande de l'écotype. Cinquante-quatre (54) produits alimentaires dérivés du maïs ont été répertoriés parmi lesquels le " owo », le " ogui » sont les plus consommés. Plusieurs écotypes de maïs sont aptes à la transformation en divers produits dérivés. Selon cette étude les plus aptes à la transformation sont les écotypes locaux. Une étude doit être menée sur la caractérisation génétique des écotypes répertoriés afin d'éliminer les probables doublons. Aussi, suggérons nous une étude sur la caractérisation physicochimique de ces écotypes et l'évaluation de leur aptitude aux diverses transformations. 


\section{REMERCIEMENTS}

Nous remercions tous les producteurs et transformatrices de maïs qui ont permis la réalisation de cette enquête. Nous remercions également le Programme Cadre d'Appui à la Diversification Agricole

\section{RÉFÉRENCES BIBLIOGRAPHIQUES}

Anonyme, 1994. Production et valorisation du maïs à l'échelon villageois en Afrique de l'Ouest. Actes de séminaire " Maïs prospère » du $25-28$ janvier 1994. CIRAD - FSA/UNB, Cotonou, Bénin, $304 \mathrm{pp}$.

Asiedu M, Nilsen R, Lie O, Lied E, 1992. Effect of processing (sprounting and /or fermentation) on sorghum and maize: Proximate composition, minerals and fatty acids. Food Chemistry 4 : 351-353.

Baco MN, Abdoulaye T, Sanogo D, Langyintuo A. 2010. Caractérisation des ménages producteurs de maïs en zone de savane sèche au Bénin. Rapport pays-Enquête-ménage - Benin. IITA Ibadan, Nigeria 38 pp.

FAO, 2015. http://fr.actualitix.com/pays/ben/beninproduction-de-cereales.php consulté le 20 janvier 2016.

Friedman J, Yaniv Z, Dafni A, Palewitch D, 1986. A preliminary classification of the healing potential of me dicinal plants, based on a rational analysis of an ethnopharmacological field survey among Bedouins in the Negev Desert, Israel. Journal of Ethnopharmacology $16: 275-287$.

Husson F, Josse J, Le S, Mazet J, 2013. FactoMineR: Multivariate Exploratory Data Analysis and Data Mining with $\mathrm{R}$. $\mathrm{R}$ package version 1.24. http://CRANR-projectorg/package=FactoMineR

Mestres C, Bada-ogoun MC, Michodjehoun L, Hounhouigan J, 2001. Compte rendu d'étude sur la « valorisation des produits vivriers au Bénin ». CIRAD-CERNA, 27pp.

Nago CM, 1989. Technologies traditionnelles et alimentation au Bénin : aspects techniques, biochimiques et nutritionnels. Cotonou, Bénin, Université Nationale du Bénin, 222pp.

Nago M, Akissoë N, Matencio F., Mestres C., 1997. End use quality of some african corn kernels. . Physico-chemical characteristics of kernels and their relationship with the quality of "Lifin", a traditional whole dry-milled maize flour from Benin. Journal of Agricultural and Food Chemistry, 45 : 555-564.
(ProCAD) par le biais du Programme de Productivité Agricole de l'Afrique de l'Ouest (PPAAO) pour le soutien financier.

Nago CM, 1997. La transformation alimentaire traditionnelle du maïs au Bénin : détermination des caractéristiques physicochimiques des variétés en usage ; relations avec l'obtention et la qualité des principaux produits dérivés. Thèse de doctorat d'Etat EsSciences, Université Paris 7-Denis Diderot- UFR de Biochimie, 199 pp.

Nout R, Hounhouigan DJ, Boeckel TV, 2003. Les aliments : transformation, conservation et qualité (Backhuys Publishers), Wageningen, Pays Bas.

OCDE, 2011. Stratégie pour une croissance verte : agriculture et agroalimentaire; Rapport préliminaire, 90p.

PRESAO, 2011. Analyse de la compétitivité du maïs local au Bénin. Résumé № 1- 2011-12 - Maïs. Décembre 2011. Programme de Renforcement et de Recherche sur la Sécurité Alimentaire en Afrique de l'Ouest.

R Core development Team, 2013. R : A language and environment for statistical computing. $R$ Foundation for Statistical Computing, Vienna, Austria ISBN3-900051-07-0. http://wwwRprojectorg/

Yallou, 2010. Répertoire des Variétés de Maïs Vulgarisées au Bénin. Dépôt légal $N^{\circ} 4920$ du 03 Décembre 2010, 4 ème trimestre, Bibliothèque Nationale (BN) du Bénin.

Yallou CG, Aïhou K, Adjanohoun A, Baco MN, Sanni OA, 2010. Itinéraires techniques de production de semences de pré base, de base et certifiées de qualité des variétés de maïs. Dépôt légal $\mathrm{N}^{\circ}$ 4921 du 03/12/2010, du 4ème trimestre, Bibliothèque Nationale (BN) du Bénin, 22 pp.

Yallou CG, Badu-Apraku B, Menkir A, 2010. Création de deux populations précoces de maïs résistantes au Striga hermonthica et tolérantes à la sécheresse. Fiche Technique. Dépôt légal $\mathrm{N}^{\circ}$ 4923 du 03/12/2010, 4ème trimestre, Bibliothèque Nationale (BN) du Bénin, 8 pp. 